\title{
Tundra arthropods provide key insights into ecological responses to environmental change
}

\author{
Toke T. Høye ${ }^{1} \mathbb{D} \cdot$ Lauren E. Culler ${ }^{2,3}$
}

Received: 18 June 2018 / Revised: 22 June 2018 / Accepted: 22 June 2018 / Published online: 3 July 2018

(c) Springer-Verlag GmbH Germany, part of Springer Nature 2018

\begin{abstract}
Arthropods perform critically important functions in food webs and ecosystems and are highly sensitive to the effects of environmental change. Despite their importance, the knowledge gaps in arthropod ecology are substantial. This is particularly problematic in Earth's polar and alpine regions, where tundra ecosystems are responding rapidly to climate change. Species diversity is lower in these regions versus temperate and tropical regions, but (1) we lack baseline and long-term data about the distributions and abundances of arthropods and their relationships to abiotic variation and (2) the roles of arthropods in these ecosystems are far from fully described. In recognition of the need for increased tundra arthropod research activity, a group of international scientists formed the Network for Arthropods of the Tundra (NeAT). In the past 3 years, this academic network has brought together entomologists from research institutions around the world to revitalize and coordinate the study of tundra arthropods. This special issue on the ecology of tundra arthropods represents a tangible example of this increased momentum. The papers in the special issue highlight recent advances in understanding the relationships between arthropod communities and abiotic variation in tundra ecosystems and clarify the roles that arthropods play in ecosystems. They collectively demonstrate the utility of tundra arthropods as a model system for testing general ecological theory about how species respond to environmental variation. We hope this special issue with the insights it provides and the new frontiers it outlines, together with NeAT, will leverage further momentum to the interest in, and study of the ecology of tundra arthropods in the years to come.
\end{abstract}

Keywords Abundance $\cdot$ Behaviour $\cdot$ Climate $\cdot$ Insects $\cdot$ Life-history variation $\cdot$ Species interactions $\cdot$ Spiders

\section{Introduction}

Global change is rapidly transforming living conditions for plants and animals on Earth (Tittensor et al. 2014). Monitoring programs are needed to track changes in the biota and the environment as these changes unfold (Pereira et al. 2010). Yet, a fundamental understanding of the ecology and

This article belongs to the special issue on the "Ecology of tundra arthropods", coordinated by Toke T. Høye and Lauren E. Culler

Toke T. Høye

tth@bios.au.dk

1 Arctic Research Centre and Department of Bioscience, Aarhus University, Grenåvej 14, 8410 Rønde, Denmark

2 Institute of Arctic Studies, Dartmouth College, Hanover, NH, USA

3 Environmental Studies Program, Dartmouth College, Hanover, NH, USA drivers of change in species, communities, and the dynamics of ecosystems is required to make such monitoring programs rigorous and focused at the same time (Lindenmayer and Likens 2018). While such knowledge is more readily available for birds, mammals, and plants, it is very fragmented for arthropods and other invertebrates. Arthropods form a major part of the global terrestrial and freshwater species diversity and include diverse taxa such as insects, collembolans, arachnids, hexapods, and freshwater crustaceans. Arthropods have very diverse life-history strategies, typically short generation times, adaptations to cope with extreme environmental conditions, and large population sizes, which make many species amenable to field studies. Despite their small size and often inconspicuous nature, these organisms play a prominent role in nutrient cycling and food web interactions, such as pollination, pest regulation, defoliation, and disease transmission. Finally, it is becoming increasingly clear that arthropods are highly sensitive to ongoing environmental 
change (Johnson and Hefin Jones 2016). Hence, a better understanding of the ecology of arthropods is needed.

Long-term observations provide a unique basis for understanding species responses to natural fluctuations in environmental conditions (Post and Høye 2013). The availability of long-term data on arthropods, however, is limited in temporal and spatial extent, and in many cases confined to coarse taxonomic resolution. This is partly due to the challenge of identifying arthropod species and the limited public interest in recording observations of more obscure taxa. Nevertheless, comparative studies often identify insects and other arthropods as more sensitive to environmental change than vertebrates and plants (Chen et al. 2011; Devictor et al. 2012; Thackeray et al. 2016; Warren et al. 2018). The limited knowledge about the ecology of most arthropod species is becoming a substantial bottleneck for identifying the underlying mechanisms of observed changes in the few monitored arthropod populations and species groups. Together, this means that we have limited ability to predict the future dynamics of one of the most climatically sensitive groups of organisms, the arthropods (Warren et al. 2018).

Tundra ecosystems in polar and alpine regions provide a unique setting for studying arthropod ecology in the context of environmental change (Høye and Sikes 2013). These locations are the fastest changing regions on Earth, with rates of temperature increase exceeding the global average, particularly in the Arctic (Overland et al. 2017) and on the Antarctic peninsula (Turner et al. 2014) where tundra is most prevalent. Warming and changing precipitation patterns are drastically affecting living conditions by affecting seasonality, hydrology, and the frequency of extreme events (e.g., Høye et al. 2007; Bokhorst et al. 2015). The distinct biogeographic patterns due to past glacial dynamics in this region offer opportunities for studying ecological disequilibrium dynamics and migrational lags (Svenning et al. 2015). Finally, tundra arthropod diversity is relatively low making species identification more tractable for non-specialists, and the low tundra vegetation makes sampling arthropod niche space simpler than elsewhere.

The study of tundra arthropods in the context of rapid environmental change has indeed yielded insights into general patterns of ecological change (Høye et al. 2013; Culler et al. 2015). Tundra arthropods exhibit significant phenological responses to these changes (Høye and Forchhammer 2008; Tulp and Schekkerman 2008; Høye et al. 2014). Their body sizes (Høye et al. 2009; Bowden et al. 2015) and abundances (Koltz et al. 2018b; Loboda et al. 2018) reflect short- and long-term changes in their abiotic and biotic environments. However, working in remote Arctic, Antarctic, and alpine locations has drawbacks. Baseline data are often lacking or entirely absent, and the first coordinated monitoring programs, such as in Zackenberg, Greenland, only began in the mid-1990s. Working in remote polar and alpine regions also requires overcoming significant logistic hurdles, which makes it near impossible for research groups to conduct comparative work across larger areas and over the longer term (Post and Høye 2013). This makes standardization of measurements and coordination among research groups particularly relevant in this region.

\section{Network for arthropods of the tundra (NeAT)}

Until recently, research efforts to address this imbalance were scattered, uncoordinated, and relied on the expertise of a few experts. In recognition of the need for increased research activity, a group of international scientists formed the Network for Arthropods of the Tundra (NeAT). This academic network on terrestrial and freshwater arthropods of Arctic, Antarctic, and alpine regions brings together entomologists from research institutions around the world. The goal of NeAT is to revitalize the study of tundra arthropods by advancing communication, collaboration, and knowledge about tundra arthropods.

As of June 2018, NeAT has 129 members from 18 countries. Members have organized and participated in numerous international conferences and symposia. NeAT working groups are currently developing protocols for multi-site comparative work and distributed experiments (Fraser et al. 2013), which will engage researchers, students, and citizen scientists. Recently, NeAT has gained status as a thematic network of the University of the Arctic (UArctic). In 2016, NeAT members gathered twice, first for planning at the Institute of Arctic Studies at Dartmouth College in the US and then for the inaugural NeAT meeting in Denmark at the Aarhus Institute of Advanced Studies, Aarhus University. The collection of papers in this issue derives from these meetings, which involved more than 50 scientists from 12 countries. They were compiled and edited by the lead and co-lead of NeAT.

Below, we present a synopsis of the papers in this issue as they relate to a range of research themes. As part of our conclusions, we provide suggestions of the role of research on tundra arthropods in developing and testing general ecological theory. The topics of the papers in this issue are representative of the diverse makeup of NeAT — scientists and scholars working at both poles and in alpine regions to understand current topics in tundra arthropod ecology. One set of papers (Docherty et al. 2018; Høye et al. 2018; Bowden et al. 2018; Convey et al. 2018; Hein et al. 2018; Turney et al. 2018) is broadly focused on characterizing how the abundance and life-history traits of tundra arthropods vary in relation to spatial and temporal environmental variation. The second set (Barrio et al. 2017; Koltz et al. 2018a; Sanchez-Ruiz et al. 2018; Urbanowicz et al. 2017; Müllerová et al. 2018) is focused on characterizing the ecological roles 
of tundra arthropods in food webs and ecosystems. We have organized the synopsis around these two broad themes.

\section{Tundra arthropods and spatio-temporal environmental variation}

A prerequisite to tracking changes in the distribution of species is to map their current distributions and identify their suitable habitats. One set of papers in this special issue expand baseline data on species presence/absence and abundance in diverse habitats (tundra, taiga, freshwater, vegetation, elevation, Arctic, Antarctic). These are extremely useful data for developing monitoring protocols as well as by identifying common approaches (e.g., pitfall traps) and indicator species. They also provide key insights about the relationships between arthropods and environmental variability, which is most essential for predicting how abundances and diversity will shift with climate change.

The results from three papers in this issue suggest focal groups of arthropods as indicators of change. Spiders and beetles are particularly useful focal groups, as they are easy to collect in a standardized manner (e.g., using pitfall traps); and Bowden et al. (2018) and Høye et al. (2018) both identified arachnid species that are associated with or responsive to changes in precipitation, soil moisture, and vegetation. For example, the spiders Arctosa insignita (Lycosidae) and Wabasso quaestio (Linyphiidae) were found to be indicators of wet, fen habitats at Narsarsuaq, South Greenland and thus are likely to be impacted by changes in hydrology associated with rapid Arctic warming (Høye et al. 2018). At the same location, three beetle species and two spider species found at low elevation were also identified as likely candidates to move into higher elevations or higher latitude as conditions change (Høye et al. 2018). Turney et al. (2018) found greater abundances of Diptera (Culicidae, Cecidomyiidae, Empididae) but lower abundances of the hemipteran family Tingidae at their tundra sites versus taiga sites in Yukon, Canada. Thus, as conditions change, flies (Diptera) and true bugs (Hemiptera) may be two additional groups to track as indicators of change.

The effects of climate change on species abundances and community composition are modulated by local environmental conditions; i.e., the effects of climate change will not be equal across the tundra. Convey et al. (2018) identify a key gap in our understanding of the microclimatic conditions experienced by tundra arthropods at soil surfaces and in the vegetation. They move beyond reiterating that such conditions can be vastly different from the standard climate data measured at weather stations two metres above the ground by providing a unique multi-annual dataset on temperatures measured at ground level from various Arctic and Antarctic sites. While they identify interesting differences and similarities in the temperature variation among Arctic and Antarctic sites, they also highlight the dramatic differences in microclimatic conditions that occur even across very small distances (Convey et al. 2018).

The notion of local variation in environmental conditions is picked up in the long-term analyses of populations of individual spider species by Bowden et al. (2018). Based on identification of almost 30,000 spider specimens collected annually at Zackenberg Northeast Greenland across an 18-year period, they show how species of spiders respond differently to environmental change in separate habitats. They also show how solar radiation, which is a proxy of soil surface temperature, is an important predictor of the activity levels of ground active spiders. Such long-term studies can help identify the most important drivers of inter-annual variation in species' abundance and points to the interesting result that the drivers of annual changes may not be equal across habitats. Similarly, in a space-for-time design, Høye et al. (2018), using lower elevation sites as a proxy for future, warmer conditions, found that arthropod composition changed with elevation but that the change was dependent on local gradients in soil moisture and vegetation. These studies, and Turney et al. (2018), highlight the importance of replicated sampling within and among habitats to accurately characterize the sometimes tight associations between arthropod species and specific habitat conditions.

Studies of life-history traits are essential and complementary to studies of species' distributions and abundance because they can provide mechanisms through which abundances vary with environmental variation. In tundra regions, environmental gradients are very pronounced and spatial and temporal variation in such life-history trade-offs become particularly evident. This is the case of the clutch size and egg-size trade-offs in wolf spiders as studied by Hein et al. (2018). The authors find that such trade-offs are more sensitive to inter-annual variation in climatic conditions than to spatial variation along the tree line in Pardosa palustris. The study highlights how the strong environmental gradients of tundra ecosystems can help better understand how the habitat template interacts with climatic variability to define key life-history trade-offs.

The relationship between species, habitats, and climate is complex and Turney et al. (2018) and Docherty et al. (2018) present results that highlight how assemblages of tundra arthropods can be distinct among sampling locations in ways that are not easily explained by environmental variability. The Turney et al. (2018) paper is an example of how studying arthropod community structure and composition along latitudinal gradients can help us predict how high-latitude communities will change as environmental conditions become more similar to those of present-day lower-latitude sites, even if no one specific driver can be identified. Docherty et al. (2018) represented freshwater 
ecosystems and presented interesting and rare result from snow-fed streams in Northeast Greenland. Building on a model for glacier-fed streams they aimed at classifying sections of streams in terms of their arthropod community composition and ecosystem properties. As a first attempt, they concluded that the glacier-fed stream model was useful, but large among stream variability calls for further sampling of snow-fed stream communities to build a more rigorous classification of streams (Docherty et al. 2018).

\section{Biotic interactions and ecosystem effects}

The second set of papers in the special issue characterizes the role arthropods play in important ecosystem process such as pollination, aquatic terrestrial linkages, nutrient cycling, predator-prey, and plant-herbivore interactions. Without understanding the roles that arthropods currently play, we lose the ability to anticipate the effects of changing species abundances and distributions with climate change. These papers also contribute important baseline data and frameworks from which we can test how changing distributions will impact food web interactions and nutrient cycling.

There is substantial attention around ongoing and predicted greening of the Arctic, but the spatio-temporal variation in tundra vegetation is not fully understood (Myers-Smith et al. 2015; Andrew et al. 2017). Biological feedback mechanisms from, e.g., insect herbivores could play an important role in this respect (Phoenix and Bjerke 2016; Lund et al. 2017). Barrio et al. (2017) and Koltz et al. (2018a), despite having vastly different research approaches, reached similar conclusions about low impacts of invertebrate herbivory, specifically direct consumption of live plant biomass, on plant damage and nutrient cycling. Barrio et al. (2017) assessed invertebrate herbivory on over 30,000 birch leaves (Betula sp.) that were collected across the Arctic using standardized protocols. Invertebrate damage was found on $11.2 \%$ of leaves but the total area damaged was only $1.4 \%$. Koltz et al. (2018a) collected samples of 33 functional feeding groups of mostly arthropods from soil, surface, and canopy habitats at a moist acidic tundra site in Alaska. From their density estimates they modelled biomass of each group to parameterize an energetic food web framework, which ultimately showed that only $0.06 \%$ of carbon processed in this food web comes from the consumption of live plant biomass.

Both of these studies provide frameworks and protocols that can be integrated and applied in other systems to understand how Arctic change will affect species interactions and nutrient cycling. In fact, Barrio et al. (2017) already demonstrated this. Their leaf samples were collected from 56 locations between 2008 and 2015. As such, they were able to discover that herbivory rates were higher in places or during years with higher summer temperatures. The energetic food web framework provided in Koltz et al. (2018a) could be used to model how nutrient cycling would change in response to this increase in herbivory, or to invertebrate herbivore outbreaks, which are not uncommon in Subarctic and tundra ecosystems (Jepsen et al. 2008; Post and Pedersen 2008). It would also be useful to parameterize the energetics model with biomass estimates from standardized sampling protocols from sites with different environmental conditions to test how nutrient cycling varies across space and time. Using samples collected from different elevations (Нøye et al. 2018) and latitudes (Turney et al. 2018) as a spatial substitute for time could generate predictions about how climate change will affect nutrient cycling through invertebrate and microbial food webs.

Pollination is another key interaction in which arthropods are the only animal group involved within tundra ecosystems. While many plant species have evolved selfing strategies or are predominantly wind pollinated, experimental studies have demonstrated a clear advantage in terms of seed set to insect pollination. In the harsh Arctic tundra of West Greenland, Urbanowicz et al. (2017) asked how important exposure to wind and proximity to the cold icecap is for the structure of pollen transfer networks. Ironically, the harsh conditions also mean that studying plant-pollinator interactions is rather time-demanding, because frequency of visits is much lower than under more benign conditions. Nevertheless, in their study, they found no effect of the landscapescale variation in temperature and wind speed on characteristics of their pollen transfer network. This is probably due to the generalistic nature of plant-pollinator interactions in the Arctic in general (e.g., Olesen et al. 2008).

One tundra arthropod family that is particularly conspicuous in the Arctic is Culicidae. Müllerová et al. (2018) focused on mapping the distribution of vector-borne diseases by screening a well-known representative species of this family, Arctic mosquitoes (Aedes nigripes), from Greenland and Svalbard, something that had not previously been done. They found interesting negative results, suggesting that Arctic island regions are not yet exposed to a number of vectorborne diseases that have been documented in continental locations (Müllerová et al. 2018). Future changes in environmental conditions and the introduction of new species or arboviruses could bring about novel species interactions that have consequences for polar wildlife and even humans.

Resource availability, which can fluctuate substantially in tundra ecosystems, is a key example of a trophic driver of population change and can elicit conspicuous behavioural responses. One of the most renowned examples of such temporally fluctuations in animal populations are the intense swarms of non-biting midges at Lake Myvatn in Iceland (Ives et al. 2008). Sanchez-Ruiz et al. (2018) found that orb-weaving spiders aggregate and build high densities of 
webs during the abrupt and intense pulse of prey availability when Chironomids emerge from Lake Myvatn. As such, this is another indication that nutrient transfer from aquatic to terrestrial ecosystems can be substantial in tundra habitats (Gratton et al. 2008).

\section{Conclusions}

Building a firm ecological understanding for such a speciose group as the arthropods of tundra habitat is no small undertaking. We welcome the recent increased interests and research activity associated with this group, and proudly acknowledge this special issue as a tangible example of this increased momentum. The collection of papers in this special issue highlight how tundra arthropods can function as model system for testing general ecological theory about how species respond to environmental variation. The lower diversity means, that it is more tractable to quantify variation in arthropod community composition across space and time, e.g., across biome transitions, where elevation works as a good proxy. Life-history trade-offs are likely to be more pronounced in the highly variable tundra environments and the influence of extreme events on life-history traits and population dynamics are easier to quantify, due to the high population sizes of individual species. Similarly, species interactions and their sensitivity to environmental change are more tractable to study as it is easier to identify and track focal species interactions.

There is a great need for new tools and methods in ecology to bridge the identified gaps in spatial and temporal resolution between what organisms respond to and what can currently be quantified (Potter et al. 2013; Estes et al. 2018). For reasons mentioned above, it is logical to develop and make the first tests of such tools in tundra ecosystems. For instance, molecular tools for species identification offer new opportunities for studying food web dynamics and temporal variation in species interactions (Wirta et al. 2015). The lower diversity makes it simpler to develop complete DNA reference libraries in tundra ecosystems (Wirta et al. 2016). Nevertheless, developing such tools require close collaboration with natural history museums to ensure that DNA libraries are accurate and up-to-date and museum collections are digitized and publically available (Sikes et al. 2017). Similarly, image-based methods hold the promise to improve temporal and spatial resolution of observations of species, biotic interactions and relationships to the environment (Weinstein 2018). Making such new tools available and operational will require substantial interdisciplinary efforts among molecular biologists, eco-physiologists, ecologists, taxonomists, engineers and computer scientists and collaboration among universities and natural history museums, something NeAT aims to facilitate.
There are still substantial holes even in the most basic ecological knowledge on arthropods. Particularly, the lack of temporal, spatial, and taxonomic resolution in available data is a central challenge for understanding species-environment relationships for tundra arthropods. In terms of temporal patterns, we lack basic knowledge about the generation time, overwintering stages, and seasonal dynamics even of the most intensively studies species. In terms of spatial patterns, we identify the extremely coarse spatial resolution of distribution information as a critical bottleneck for tracking contemporary range changes and understanding the historical background for biogeographic patterns and population genetic variation. At smaller spatial scale, we are also only beginning to understand the habitat specificity of individual species. Finally, the taxonomic resolution in studies of tundra arthropod populations is often limited. Many datasets have order- or family-level resolution or are confined to wellknown groups (e.g., spiders, beetles or butterflies) where identification by morphological characters requires less specialized expertise. Because of these deficiencies, red lists for Arctic arthropods are nowhere near complete, which would form the basis for targeted conservation actions. Although many Arctic arthropod species appear widespread, they are likely to suffer substantial range contractions as species from lower latitudes expand their ranges northwards (Eskildsen et al. 2013). Finally, we are only just beginning to grasps the intricate species-specific responses to environmental change, the implications for food web dynamics and the detailed biotic interactions in which arthropods play a role (Schmidt et al. 2017).

Many of knowledge gaps listed above apply to arthropod ecology in other biomes as well. It is an ambition of $\mathrm{NeAT}$ to build on the public interest in climate change and its biological consequences particularly in the Arctic to help raise awareness of the intricate adaptations and fascinating ecology of this group of organisms anywhere on the planet. Building strong collaboration with students, teachers and schools in northern community is of critical importance in this endeavour and we envision that clear and exciting protocols for sampling and studying arthropods will determine the success of such efforts. There are many models to build on such as the Teatime4Science (http://www.teatime4science .org/) and the School Malaise Trap Project (https://malai seprogram.com/). Even at northern latitudes, such protocols and smartphone apps are beginning to emerge for example to engage the public in mapping the northward spread of forest pest insects in the Moth Hunt (https://www.malerjakt.no/). Similarly, there are national monitoring initiatives such as the National Ecological Observatory Network (https://www. neonscience.org/) and Greenland Ecosystem Monitoring (www.g-e-m.dk), and international Arctic initiatives such as the Circumpolar Biodiversity Monitoring Programme (https ://www.caff.is/monitoring) to learn from and collaborate 
with. We hope this special issue with the insights it provides and the new frontiers it outlines, together with NeAT, will leverage further momentum to the interest in and study of the ecology of tundra arthropods in the years to come.

Acknowledgements We thank the Danish Agency for Science, Technology and Innovation for supporting this work with an International Network Programme Grant (5132-00144B) to TTH. The Institute of Arctic Studies at Dartmouth College's John Sloan Dickey Center for International Understanding kindly provided meeting facilities during the planning workshop. The Arctic Research Centre and the Aarhus Institute of Advanced Studies at Aarhus University are kindly acknowledged for financial support of the 1 st NeAT meeting. We thank the editor in chief at Polar Biology for excellent support and all the reviewers for their help during the preparation of this special issue.

\section{References}

Andrew CM, Elizabeth SJ, Gillian P, Isla M-S, Marc M-F (2017) Shrub growth and expansion in the Arctic tundra: an assessment of controlling factors using an evidence-based approach. Environ Res Lett 12:085007

Barrio IC et al (2017) Background invertebrate herbivory on dwarf birch (Betula glandulosa-nana complex) increases with temperature and precipitation across the tundra biome. Polar Biol 40:2265-2278. https://doi.org/10.1007/s00300-017-2139-7

Bokhorst S, Phoenix GK, Berg MP, Callaghan TV, Kirby-Lambert C, Bjerke JW (2015) Climatic and biotic extreme events moderate long-term responses of above- and belowground sub-Arctic heathland communities to climate change. Glob Change Biol 21:4063-4075. https://doi.org/10.1111/gcb.13007

Bowden JJ, Eskildsen A, Hansen RR, Olsen K, Kurle CM, Høye TT (2015) High-Arctic butterflies become smaller with rising temperatures. Biol Lett 11:20150574. https://doi.org/10.1098/ rsbl.2015.0574

Bowden JJ, Hansen OLP, Olsen K, Schmidt NM, Høye TT (2018) Drivers of inter-annual variation and long-term change in High-Arctic spider species abundances. Polar Biol. https://doi.org/10.1007/ s00300-018-2351-0

Chen IC, Hill JK, Ohlemuller R, Roy DB, Thomas CD (2011) Rapid range shifts of species associated with high levels of climate warming. Science 333:1024-1026. https://doi.org/10.1126/scien ce. 1206432

Convey P, Coulson SJ, Worland MR, Sjöblom A (2018) The importance of understanding annual and shorter-term temperature patterns and variation in the surface levels of polar soils for terrestrial biota. Polar Biol. https://doi.org/10.1007/s00300-018-2299-0

Culler LE, Ayres MP, Virginia RA (2015) In a warmer Arctic, mosquitoes avoid increased mortality from predators by growing faster. Proc R Soc B 282:20151549. https://doi.org/10.1098/ rspb.2015.1549

Devictor V et al (2012) Differences in the climatic debts of birds and butterflies at a continental scale. Nat Clim Change 2:121-124. https://doi.org/10.1038/Nclimate1347

Docherty CL, Hannah DM, Riis T, Leth SR, Milner AM (2018) Longitudinal distribution of macroinvertebrates in snowmelt streams in northeast Greenland: understanding biophysical controls. Polar Biol. https://doi.org/10.1007/s00300-017-2212-2

Eskildsen A et al (2013) Testing species distribution models across space and time: high latitude butterflies and recent warming. Global Ecol Biogeogr 22:1293-1303. https://doi.org/10.1111/ geb. 12078
Estes L et al (2018) The spatial and temporal domains of modern ecology. Nat Ecol Evol 2:819-826. https://doi.org/10.1038/s4155 9-018-0524-4

Fraser LH et al (2013) Coordinated distributed experiments: an emerging tool for testing global hypotheses in ecology and environmental science. Front Ecol Environ 11:147-155. https://doi. org/10.1890/110279

Gratton C, Donaldson J, Zanden MJV (2008) Ecosystem linkages between lakes and the surrounding terrestrial landscape in Northeast Iceland. Ecosystems 11:764-774. https://doi.org/10.1007/ s10021-008-9158-8

Hein N, Brendel MR, Feilhauer H, Finch O-D, Löffler J (2018) Egg size versus egg number trade-off in the alpine-tundra wolf spider, Pardosa palustris (Araneae: Lycosidae). Polar Biol. https://doi. org/10.1007/s00300-018-2301-x

Høye TT, Forchhammer MC (2008) Phenology of High-Arctic arthropods: effects of climate on spatial, seasonal and inter-annual variation. Adv Ecol Res 40:299-324. https://doi.org/10.1016/S0065 -2504(07)00013-X

Høye TT, Sikes DS (2013) Arctic entomology in the 21st century. Can Entomol 145:125-130. https://doi.org/10.4039/Tce.2013.14

Høye TT, Post E, Meltofte H, Schmidt NM, Forchhammer MC (2007) Rapid advancement of spring in the High Arctic. Curr Biol 17:R449-R451

Høye TT, Hammel JU, Fuchs T, Toft S (2009) Climate change and sexual size dimorphism in an arctic spider. Biol Lett 5:542-544

Høye TT, Post E, Schmidt NM, Trøjelsgaard K, Forchhammer MC (2013) Shorter flowering seasons and declining abundance of flower visitors in a warmer Arctic. Nat Clim Change 3:759-763. https://doi.org/10.1038/Nclimate1909

Høye TT, Eskildsen A, Hansen RR, Bowden JJ, Schmidt NM, Kissling WD (2014) Phenology of High-Arctic butterflies and their floral resources: species-specific responses to climate change. Curr Zool 60:243-251

Høye TT, Bowden JJ, Hansen OLP, Hansen RR, Henriksen TN, Niebuhr A, Skytte MG (2018) Elevation modulates how Arctic arthropod communities are structured along local environmental gradients. Polar Biol. https://doi.org/10.1007/s00300-017-2204-2

Ives AR, Einarsson A, Jansen VAA, Gardarsson A (2008) High-amplitude fluctuations and alternative dynamical states of midges in Lake Myvatn. Nature 452:84-87. https://doi.org/10.1038/natur e 06610

Jepsen JU, Hagen SB, Ims RA, Yoccoz NG (2008) Climate change and outbreaks of the geometrids Operophtera brumata and Epirrita autumnata in subarctic birch forest: evidence of a recent outbreak range expansion. J Anim Ecol 77:257-264. https://doi.org/10.111 $1 / \mathrm{j} .1365-2656.2007 .01339 . x$

Johnson SN, Hefin Jones T (2016) Introduction to global climate change and terrestrial invertebrates. In: Johnson SN, Hefin Jones $\mathrm{T}$ (eds) Global climate change and terrestrial invertebrates. Wiley, Hoboken, pp 1-8. https://doi.org/10.1002/9781119070894.ch1

Koltz AM, Asmus A, Gough L, Pressler Y, Moore JC (2018a) The detritus-based microbial-invertebrate food web contributes disproportionately to carbon and nitrogen cycling in the Arctic. Polar Biol. https://doi.org/10.1007/s00300-017-2201-5

Koltz AM, Schmidt NM, Høye TT (2018b) Differential arthropod responses to warming are altering the structure of Arctic communities. R Soc Open Sci. https://doi.org/10.1098/rsos.171503

Lindenmayer DB, Likens GE (2018) Effective ecological monitoring, 2nd edn. CSIRO, Canberra, p 224

Loboda S, Savage J, Buddle CM, Schmidt NM, Høye TT (2018) Declining diversity and abundance of High Arctic fly assemblages over two decades of rapid climate warming. Ecography 41:265-277. https://doi.org/10.1111/ecog.02747

Lund M, Raundrup K, Westergaard-Nielsen A, López-Blanco E, Nymand J, Aastrup P (2017) Larval outbreaks in West Greenland: 
instant and subsequent effects on tundra ecosystem productivity and CO2 exchange. Ambio 46:26-38. https://doi.org/10.1007/ s13280-016-0863-9

Müllerová J et al (2018) No indication of arthropod-vectored viruses in mosquitoes (Diptera: Culicidae) collected on Greenland and Svalbard. Polar Biol. https://doi.org/10.1007/s00300-017-2242-9

Myers-Smith IH et al (2015) Climate sensitivity of shrub growth across the tundra biome. Nat Clim Change 5:887-891. https:// doi.org/10.1038/Nclimate2697

Olesen JM, Bascompte J, Elberling H, Jordano P (2008) Temporal dynamics in a pollination network. Ecology 89:1573-1582

Overland JE et al. (2017) Surface air temperature In: Arctic Report Card 2017. https://www.arctic.noaa.gov/Report-Card

Pereira HM et al (2010) Global biodiversity monitoring. Front Ecol Environ 8:459-460. https://doi.org/10.1890/10.WB.23

Phoenix GK, Bjerke JW (2016) Arctic browning: extreme events and trends reversing arctic greening. Glob Change Biol 22:2960-2962. https://doi.org/10.1111/gcb.13261

Post E, Høye TT (2013) Advancing the long view of ecological change in tundra systems. Philos Trans R Soc B 368:20120477. https:// doi.org/10.1098/Rstb.2012.0477

Post E, Pedersen C (2008) Opposing plant community responses to warming with and without herbivores. Proc Natl Acad Sci USA 105:12353-12358. https://doi.org/10.1073/pnas.0802421105

Potter KA, Arthur WH, Pincebourde S (2013) Microclimatic challenges in global change biology. Glob Change Biol 19:2932-2939. https://doi.org/10.1111/gcb.12257

Sanchez-Ruiz JA, Phillips JS, Ives AR, Gratton C (2018) Responses of orb-weaving spider aggregations to spatiotemporal variation in lake-to-land subsidies at Lake Mývatn, Iceland. Polar Biol. https ://doi.org/10.1007/s00300-017-2202-4

Schmidt NM et al (2017) Interaction webs in arctic ecosystems: determinants of arctic change? Ambio 46:12-25. https://doi. org/10.1007/s13280-016-0862-x

Sikes DS et al (2017) The value of museums in the production, sharing, and use of entomological data to document hyperdiversity of the changing North. Arctic Sci 3:498-514. https://doi.org/10.1139/ as-2016-0038
Svenning JC, Eiserhardt WL, Normand S, Ordonez A, Sandel B (2015) The influence of paleoclimate on present-day patterns in biodiversity and ecosystems. Annu Rev Ecol Evol Syst 46:551-572. https ://doi.org/10.1146/annurev-ecolsys-112414-054.314

Thackeray SJ et al (2016) Phenological sensitivity to climate across taxa and trophic levels. Nature 535:241-245. https://doi. org/10.1038/nature 18608

Tittensor DP et al (2014) A mid-term analysis of progress toward international biodiversity targets. Science 346:241-244. https://doi. org/10.1126/science. 1257484

Tulp I, Schekkerman H (2008) Has prey availability for arctic birds advanced with climate change? Hindcasting the abundance of tundra arthropods using weather and seasonal variation. Arctic 61:48-60

Turner J et al (2014) Antarctic climate change and the environment: an update. Polar Rec 50:237-259. https://doi.org/10.1017/S0032 247413000296

Turney S, Altshuler I, Whyte LG, Buddle CM (2018) Macroinvertebrate and soil prokaryote communities in the forest-tundra ecotone of the Subarctic Yukon. Polar Biol. https://doi.org/10.1007/ s00300-018-2330-5

Urbanowicz C, Virginia RA, Irwin RE (2017) The response of pollentransport networks to landscape-scale climate variation. Polar Biol 40:2253-2263. https://doi.org/10.1007/s00300-017-2138-8

Warren R, Price J, Graham E, Forstenhaeusler N, VanDerWal J (2018) The projected effect on insects, vertebrates, and plants of limiting global warming to $1.5^{\circ} \mathrm{C}$ rather than $2{ }^{\circ} \mathrm{C}$. Science $360: 791-795$. https://doi.org/10.1126/science.aar3646

Weinstein BG (2018) A computer vision for animal ecology. J Anim Ecol 87:533-545. https://doi.org/10.1111/1365-2656.12780

Wirta HK et al (2015) Exposing the structure of an Arctic food web. Ecol Evol 5:3842-3856. https://doi.org/10.1002/ece3.1647

Wirta HK et al (2016) Establishing a community-wide DNA barcode library as a new tool for arctic research. Mol Ecol Resour 16:809822. https://doi.org/10.1111/1755-0998.12489 